Aus dem Anatomischen Institut der Med. Fakultät, Univ. Okayama

(Vorstand: Prof. M. SEKI).

\title{
Beobachtung der Veränderungen der Retikulumzellen in der Schleimhaut des Wurmfortsatzes bei der Ent- zündung.
}

\author{
炎症に於ける虫垂粘膜の細網細胞の変化の追跡.
} Tetsuo SHINDO 進藤 哲 雄.

(Eingegangen am. 26. Juli 1954.)

Die Mesenchymzellen erzeugen in der Embryonalzeit sehr verschiedene Zellen. Die in dem Knochenmark und den lymphatischen Geweben gebliebenen, gleich gestalteten Retikulumzellen liefern verschiedene Blutzellen. Es ist auch bekannt, daß die Fibrocyten, welche sich in der Haut und im Bindegewebe in und zwischen den Organen des ganzen Körpers befinden, sich durch Reize bald in Histiocyten oder Monocyten, bald in leukocytenähnliche Zellen umwandeln können. Über die Umwandlungsfähigkeit der Retikulumzellen in der Schleimhaut Jiegen aber nur wenige systematische Untersuchungen vor. SEKI $(1941,1953)$ hat darauf aufmerksam gemacht, daß sich die Retikulumzellen und Fibrocyten in der menschlichen Nasenschleimhaut durch den Reiz von Gift, Virus und Bakterien in Histiocyten, Monocyten und grobgranulierte Leukocyten umwandeln können. MIYAMOTO (1954) beobachtete auch die Entstehung der genannten Zellen aus den Retikulumzellen in der durch Arzneimittel gereizten Schleimhaut der Harnblase des Kaninchens.

Ich untersuchte die frühzeitigen Veränderungen der Retikulumzellen in der Schleimhaut des Wurmfortsatzes bei der akuten Entzündung, die sich gewöhnlich durch einen sehr schnellen Fortschritt des Prozesses charakterisiert. Der Prozess beginnt an dem apikalen, mitteren oder Wurzelteil des Wurmfortsatzes und dehnt sich auf eine oder auf beide Seiten aus, so daß man an ein und demselben Material Veränderungen der Schleimhaut von verschiedener Stärke vergleichend beobachten kann.

\section{Material und Methode.}

Es wurden 62 Wurmfortsätze in der Frühzeit der Entzündung untersucht, aber auch daneben 5 normale. Die sorgfältig ausgenommenen Wurmfortsätze wurden unter Umsłänden der Länge nach geöffnet und in 10\% ige Formalinlösung gebracht. Aus jedem Wurmfortsatz nahm man 4-9 Stückchen, bettete sie in Celloidin ein, um zur Schleimhautoberfläche vertikale und parallele Schnitte von 15 « Dicke zu bekommen. 
Die Auszugserien der Schnitte wurden nach der Eisenhämateinlackmethode und mit Hämatoxylin und Eosin, sowie mit Hamätoxylin und Azur IIEosin gefärbt.

\section{Ergebnisse der Untersuchung.}

Ls gibt verschiedene Formen der Wurmfortsatzentzündung. Die Initialentzündung entwickelt sich zuweilen schnell bis zur Nekrose, bleibt in anderen Fällen katarrhalisch oder verläult sehr schleichend, zieht sich aber manchmal auch über eiren langen Zeitraum hin. Im folgenden wird die Entzündung hauptsächlich nach KIMOTO (1938) in verschiedene Gruppe eingeteilt, wobei die Ëinteilung von HIJIKATA (1935) bezüglich der frühzeitigen Veränderungen der Entzündung berücksichtigt werden. Die Abgrenzung des noch gesunden Teiles des Wurmfortsatzes gegen den Entzündungsherd trat in allen Fällen in den Vordergrund des Interesses.

\section{a) Retikulumzellen.}

Bevor wir auf die Veränderungen der Retikulumzellen in der Lamina propria mucosae bei der Wurmfortsa1zentzündung eingehen, seien die normalen Zellenbilder besprochen. Wie in Abb. 1 dargestellt ist, sind

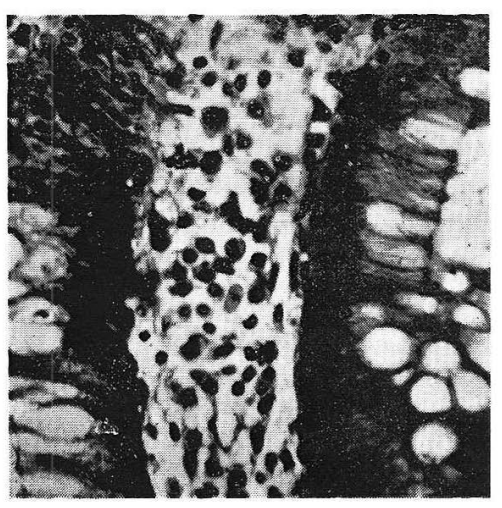

Abb. 1. Aus der Schleimhaut des mormalen Wurwfortsatzes. Man beachte die Retikulumzellen mit Ausläufern. Nach der Eisenhämateinlackmethode. $240 \times$

die Bindegewebsfasern hier ganz arm, und etwa die Hälfte der Zellen gehört zu den Retikulumzellen mit distinkt erkennbaren Auslä:ıfern. Die andere Hälfte sind kleine Lymphocyten mit schmalem saumartigem Cytoplasma sowie größere Monocyten und roch wrößere Histiocyten. Anderweitige Zellarten kommen in geringerer Zahl vor.

Schon im Anfangsstadium der katarrhalischen Wurmfortsatzentzündung, in welchem man nur wenige neutrophile polymorphkernige Leukocyten im Lumen des Wurmfortsatzes, aber keine starke pathologische Vesänderungen in der Schleimhaut sieht, fangen hier und dort die Retikulumzellen an, die Ausläufer zusammenzuziehen urd sich etwas zu vergrößern (Abb. 2). Veränderungen bis hierher werden auch in dem als normal angesehenen Wurmfortsatz häufig beobachtet. Wenn die Epithelzellen sich weitgehend verändern und ablösen, ziehen die 
meisten Retikulumzellen ihre Ausläufer zusammen und runden sich ab. Sie wandeln sich in Monocyten (Abb. 3). Die Monocyten können verschiedene Granula aufspeichern und sich bekanntlich in größere Histiocyten übergehen.

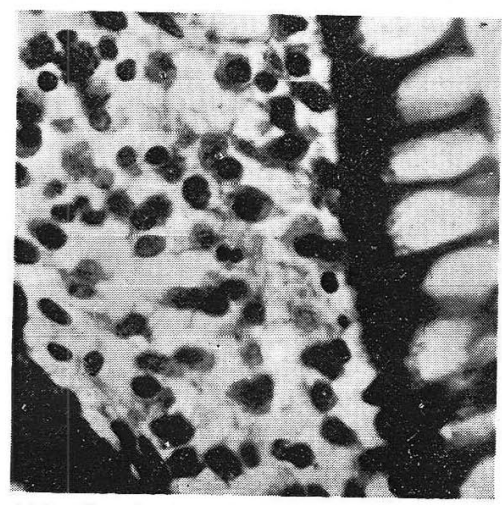

Abb. 2. Aus der Schleimhaut in der Frühzeit der katarrhalischen Entzündung. Man beachte die Abrundung der Retikulumzellen. Nach der Eisenhämateinlackmethode. $600 \times$

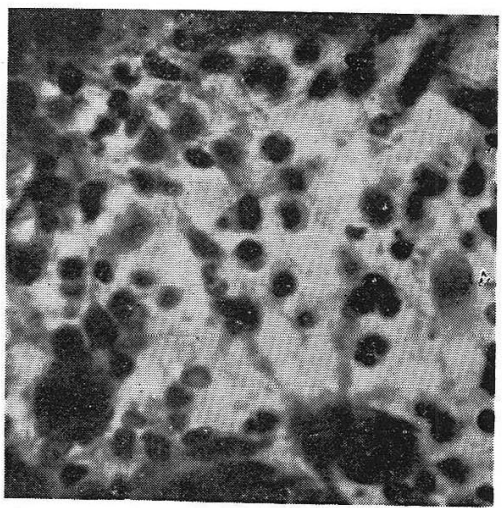

Abb. 3. Schleimhaut bei der katarrhalischen Wurmfortsatzentzündung. Monocyten vermehren sich. Nach der Eisenhämteiniackmethode. $600 \times$

Die Zahıl der Lymphocyten verändert sich nicht sehr, weil zwar viele einerseits degenerieren und zerfallon, aber zugleich neue auftreten. Ein Teil der Lymphocyten scheint, sich in monocytäre Formen umzuwandeln, aber keineswegs sehr viel. Histiccyten sind in großer Zahl zu sehen. Die polymorphkernigen Leukocyten sind bei weitem weniger, als man es erwarten kännte.

Weil solche katarrhalisehe Veränderungen auch bei der heftigen empyematösen, phlegmonös-ulcerösen und gangrenösen Wurmfortsatzentzündung an den von dem Entzündungsherd weit entfernten Orten auftreten, kann die katarrhalische Entzündung als die Vorstufe der genannten heftigen Veränderungen angesehen werden.

Die Beobachtung eines stark veränderten Entzündungsherdes selbst ist nicht der Zweck unserer Arbeit, aber ich möchte einen Blick darauf werfen. In dem Herd oder in der unmittelbaren Nähe eines solchen Herdes machen manchmal Histiocyten den größeren Teil der Zellen aus (wie in Abb. 4, ein Bild, in der Nähe der vorgeschrittenen katarrhalischen Entzündung), drängen sich andermals die Monocyten und IIistiocyten aneinander (Abb. 5, ein Bild, in der Nähe der gangrenösen Entzündung), wobei die typischen Retikulumzellen kaum mehr vorhanden sind.

\section{b) Monocyten.}

Die Monocyten treten in der Schleimhaut des normalen Wurmfort- 
satzes zerstreut auf. Bei der Entzündung geht die Monocytenvermehrung mit der Kontraktion der Ausläufer und der Abrundung des Zelleibes der Retikulumzellen parallel. Die Monocyten scheinen auch aus den Lymphocyten zu entstehen, aber die Möglichkeit ist je nach den Umständen verschieden. In der Umgebung der heftigen Entzündung erscheinen die Monocyten zahlreich (Abb. 5).

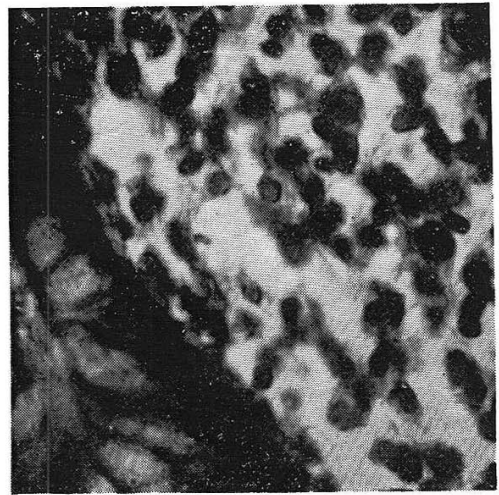

Abb. 4. Aus der Umgebung der fortgeschrittenen katarrhalischen Wurmfortsatzentzündung. Viele Histiocyten. Nach der Eisenhämateinlackmethode. $600 x$

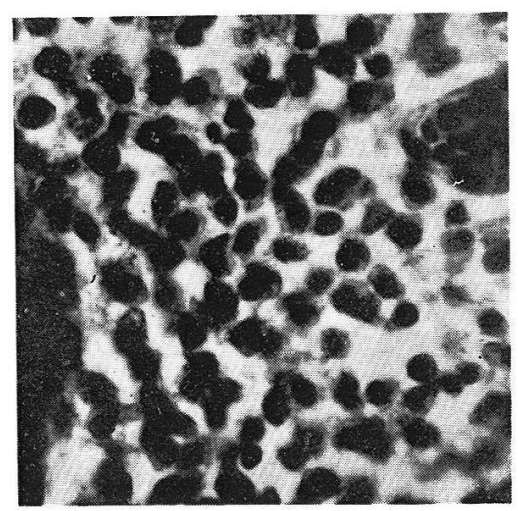

Abb. 5. Aus der Nähe der gangrenösen Entzündung des Wurmfortsatzes. Die Monocyten und Histiocyten sind gedrängt vorhanden. Nach der Eisenhämateinlackmethode. $600 \times$

Die Plasmazellen wurden im vorigen Jahrhurdert als solche definiert. Dabei hatten wir noch fast keine Ahnung von den Monocyten. Man spricht of $t$ von dem Vorkommen zahlreicher Plasmazellen in der mormalen Darmschleimhaut und von der Vermehrung derselben bei der Entzündung. Weil es aber viele den Plasmazellen ähnliche Monocyten gibt, scheinen die Monocyten häufig zu den Plasmazellen gerechnet zu werden.

\section{c) Histiocyten.}

Die Histiocyten sind in der Lamina propria mucosae der normalen Schleimhaut wenig zu sehen, aber bei der Fntzündung entstehen sie entweder unmittelbar aus den Retikulumzellen oder durch Monocyten. Sie vergrößern sich allmälich und schließen Granula und Vakuolen ein. Sie kommen auch in der Nähe der akuten Fntzündung vor (Abb. 6), aber besonders zahlreich um die chronischen Lntzündung (Abb. 7, cin Bild, nahe dem heilenden Geschwur).

\section{d) Grobgranulierte Leukocyten.}

Über die grobgranulierten Teukocyten in der Nasenschleimhaut des Menschen ist eine Untersuchung von SEKI (1941, 1953) vorhanden. Über ihr Auftreten in der Schleimhaut hatte NAGANUMA (1950) experi- 


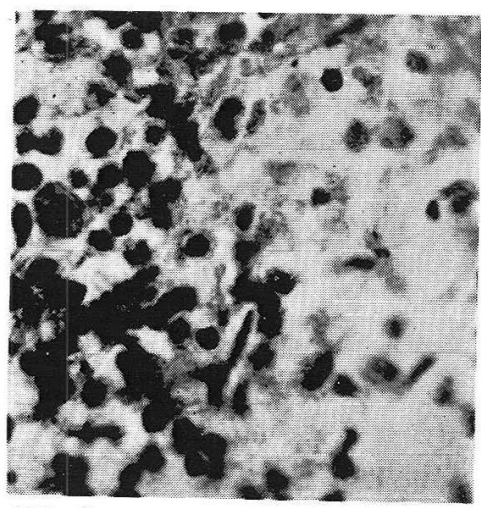

Abb. 6. Aus der Nähe der akuten Entzündung des Wurmfortsatzes. Man sieht die Monocyten und Histiocyten. Nach der Eisenhämateinlackmethode. $600 x$

mentell untersucht. Sie scheinen nach ihnen auch meistens aus den Retikulumzellen zu stammen. Die grobgranulierten Leukocyten werden auch in der Schleimhaut des normalen Darmes häufig angetroffen. Sie kommen zahlreich bei der chronischen Wurmfortsatzentzündung und in Gem Stadium der Granulationbildung vor. Sie gestalten sich, wie Abb. 8 gezeigt, rund oder oval und sind von verschiedener Größe. Sie sind manchmal zwei- bis dreimal größer als die eosinophilen Leukocyten im Blut. Die in ihrem Körper eingeschlossenen eosinophilen Granula können auch verschieden groß sein. Der Zellkern ist kugelig, nierenförmig oder zweilappig.

\section{Zusammenfassung.}

1. Die Schleimhaut des Wurmfortsatzes wird, wie die der anderen Darmabschnitte, durch chemische und biologische Substanzen und Bakterien leicht angegriffen, so daß in ihr ein Apparat vorhanden sein muß, um sie zurückzuhalten oder sie harmlos zu machen. Man sieht in der Schleimhaut Retikulumzellen, die denjenigen im Lymphsinus des Lymphknotens ähneln. Auch in der normalen Wurmfortsatzschleimhaut sind 
zahlreiche Lymphocyten und wenigere Monocyten und Histiocyten vorhanden. Der größere Teil der letzteren ist wahrscheinlich aus den Retikulumzellen entstanden.

2. Bei der katarrhalischen Wurmfortsatzentzündung werden zunächst Übergänge von den Retikulumzellen in die Monocyten gesehen, welch letztere sich später zu größere Histiocyten umwandeln können. In der Nähe der starken Entzündung geschehen auch die erwähnten Umbildungen der Zellen.

3. In der chronischen Wurmfortsatzentzündung vermehren sich die grobgranulierten Leukocyten von verschiedener Größe. Die von ihnen eingeschlossenen, mit Eosin gut färbbaren Granula sind verschieden groß. Nach anderweitigen Beobachtungen scheinen die Zellen auch meistens aus den Retikulumzellen zu stammen.

\section{內 容自 抄。}

虫垂の粘朕は腸の他の部分の粘膜と同様と種々の化学的と生物的の物質 と微生物の侵入を受け易く，従って上皮下にはこれ等を無害にしたり，捕 捉したりする装置がなくてはならない，そこでリンパ節のリンパ洞と見る 細網細胞に似た細胞があり，ここでも網網細胞と呼ばれる，健常の虫垂粘 膜にも細網細胞飞混じて多くのリンパ球と少数の単球と組織球があるが, それ等の大部分は多分細網細胞から生じたものである.

カタール性虫垂炎には先ず細網細胞の単球に変態する像が多く見られ, 単球は後に肥大して組織球になり得る。強い炎症の附近にも大体上記の細 胞変化が行われる

虫垂炎が慢性となればエオンンによく染る粒子を持つ種々の大さの粗大 粒子球が増加する。その粒子の大さ種々である，他の観察でこの細胞も 多くは細網細胞に由来することが知られている。

\section{Literatur.}

Hijikata, H.: Beiträge zur Kenntnis der mikroskopischen früzeitigen Veränderungen bei der akuten Wurmfortsatzentzündung. (Jap.) Verh. jap. chir. Ges. 35 (1935). - Kimoto, S. : Pathologische und bakteriologische Studien über die Wurmfortsatzentzündung. (Jap.) Verh. jap. chir. Ges. 39 (1938). - Miyamoto, J. : Experimentelle Untersuchung über die Umwandlungsfähigkeit der Retikulumzellen und Fibrocyten in der Schleimhaut der Harnblase des Kaninchens. Arch. hist. jap. 6 (1954). - Naganuma, K. : Über die der Nasenallergie der Maus auftretenden eosinophilen Zellen. (Jap. m. dtsch. Z-fass.) Arch. hist. jap. 1 (1950). - Seki, M. : Retikulumzellen und ihre Abkömmlinge in der Nasenschleimhaut des Menschen. Z. Zellforsch. 31 (1941); Arch. hist. jap. 4 (1953). 